\title{
What is normal? A central question in the application of CMR mapping techniques
}

\author{
Andreas A. Kammerlander · Julia Mascherbauer
}

(C) Springer-Verlag GmbH Austria, part of Springer Nature 2019

New techniques in cardiovascular imaging are rapidly evolving and unfold new pathways in clinical practice. In cardiovascular magnetic resonance imaging (CMR) T1-mapping has revolutionized the understanding of the myocardial extracellular space and its importance in a variety of cardiac disorders.

Bearing in mind the basic principles of CMR, the T1 time represents the longitudinal relaxation time and is a measure of how quickly the net magnetization vector recovers to its ground state after an impulse. The technology of T1-mapping enables visualization and quantification of the specific T1 time of every voxel within a certain region of interest, which differs between normal myocardium and pathologies, such as fibrosis, edema and infiltrative disorders.

While the well-established late gadolinium enhancement (LGE) method allows visualization of focal fibrosis, e.g. after myocardial infarction, T1-mapping additionally allows insights into more diffuse alterations of the myocardium. Using T1-mapping both before and after gadolinium administration furthermore enables the quantification of extracellular volume, which has been validated against myocardial biopsies [1-4]. So far, T1-mapping techniques have found their way into clinical practice in the diagnosis of infiltrative disorders, such as cardiac amyloidosis or Anderson-Fabry disease [5, 6] and are being investigated as risk factor in numerous clinical scenarios. Fig. 1 depicts a typical clinical application of T1mapping in patients presenting with left ventricular hypertrophy. Both cine and LGE imaging did not provide conclusive information in all three patients; however, T1-mapping revealed moderately elevated

a.o. Univ. Prof. Dr. J. Mascherbauer, MD $(\bowtie)$

Division of Cardiology, Medical University of Vienna,

Waehringer Gürtel 18-20, 1090 Vienna, Austria

julia.mascherbauer@meduniwien.ac.at
T1 times in patient 1 (990 ms, first column) with hypertrophic cardiomyopathy whereas in patient $2 \mathrm{~T} 1$ times of the myocardium were significantly lowered (840 ms, second column) prompting the suspicion of Anderson-Fabry disease, which was later confirmed by further testing. In patient 3 (third column) both native $\mathrm{T} 1$ times and the extracellular volume were markedly elevated (1190ms and $65 \%$, respectively) which is highly indicative of cardiac amyloidosis. The diagnosis was confirmed by positive enhancement in ${ }^{99} \mathrm{mTc}$-3.3-diphosphono-1.2-propanodicarboxylic acid $\left({ }^{99} \mathrm{mTc}-\mathrm{DPD}\right)$ scintigraphy indicating the presence of the transthyretin (TTR) subtype.

More recently, T2-mapping was introduced as a new method for detection of myocardial edema in addition to standard short-tau inversion recovery sequences, comparable to the value of T1-mapping on top of LGE imaging. Both T1-mapping and T2mapping are currently being considered to replace the Lake Louise criteria for the diagnosis of myocarditis $[7,8]$.

Although they harbor a unique potential for noninvasive myocardial characterization, mapping techniques are limited by the lack of reference values. This is of particular interest since the possibility of CMR T1 and T2-mapping increases referrals for CMR scans, and referral indications are shifting. Non-academic outpatient radiology centers will also increasingly be asked to report $\mathrm{T} 1$ and $\mathrm{T} 2$-mapping results as well as extracellular volume estimates of the myocardium; however, the current consensus statement of the Society of Cardiac Magnetic Resonance Imaging underlines the importance of validation of reference values in every single center [9]. Figure 1 furthermore illustrates another development of CMR: while cine and LGE imaging are mainly assessed qualitatively, T1-mapping only provides conclusive information if 
Fig. 1 T1-mapping results in three patients presenting with left ventricular hypertrophy. T1 times were $990 \mathrm{~ms}$ in the patient with hypertrophic cardiomyopathy (HCM, column 1), $840 \mathrm{~ms}$ in the patient with Anderson-Fabry disease (M. Fabry, column 2) and $1190 \mathrm{~ms}$ in the patient suffering from cardiac amyloidosis (Amyloidosis, column 3)

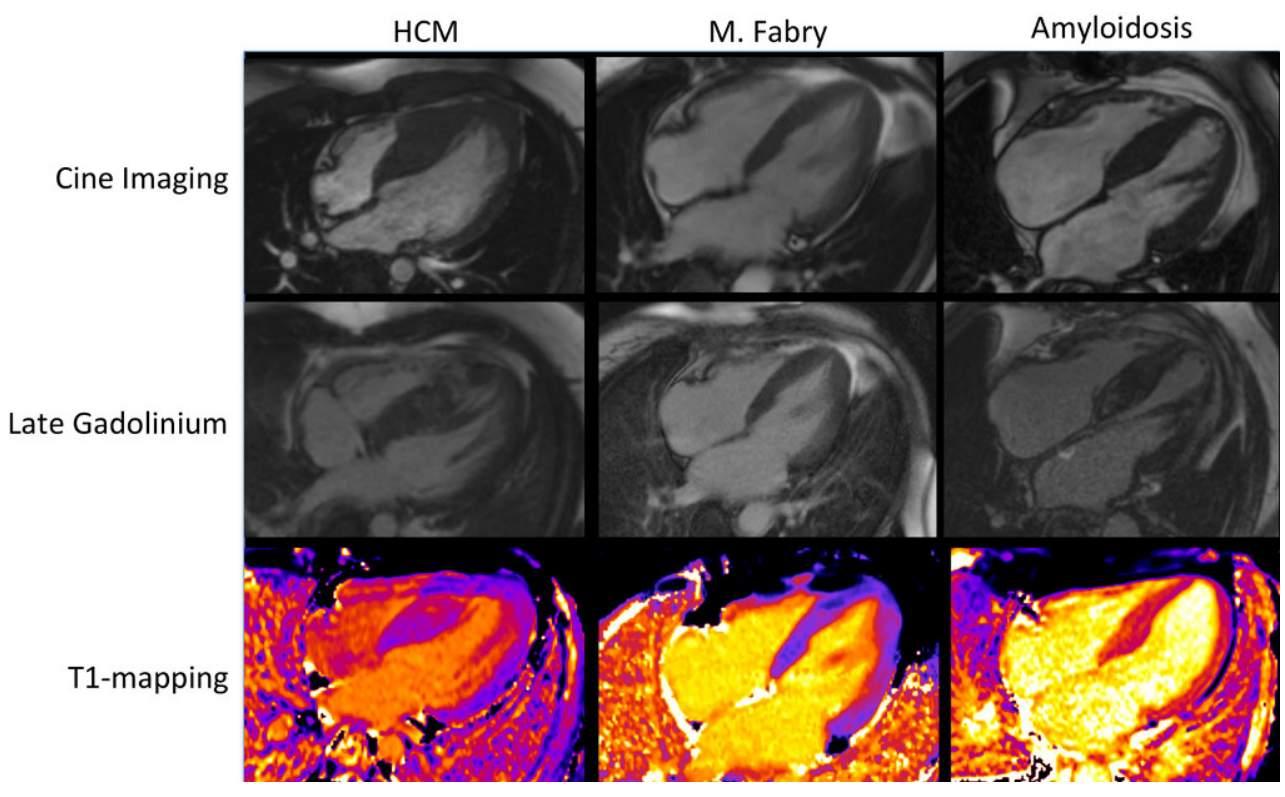

quantitative measurements are interpreted with respect to locally acquired normal values.

In this issue of the journal, Granitz et al. [10] report $\mathrm{T} 1$ and T2-mapping results of 60 healthy individuals undergoing CMR both on 1.5T and 3.0T systems. Consistent with previous findings, gender and heart rate dependency were reported for both $\mathrm{T} 1$ and $\mathrm{T} 2$ times. Reference values that are applicable in an older population are still a matter of debate. In this study, as in previous investigations, volunteers were young (mean age 41 years) and only 4 individuals were 60 years or older. Nevertheless, these data are of great importance not only for the center where they have been determined and thus enable the interpretation of local T1 maps. They will in the future, as part of a big puzzle, enhance our understanding of the variation of such measurements and the factors that determine local differences. The use of CMR mapping techniques has already revolutionized cardiovascular imaging and will continue to do so. The availability of reference values that do not need validation in every single CMR center will hence be crucial for the correct application of this innovative technique.

Conflict of interest A.A. Kammerlander and J. Mascherbauer declare that they have no competing interests.

\section{References}

1. Kammerlander AA, Duca F, Binder C, et al. Extracellular volume quantification by cardiac magnetic resonanceimaging without hematocrit sampling: Ready for prime time? Wien Klin Wochenschr. 2018;130:190-6.

2. aus dem Siepen F, Buss SJ, Messroghli D, etal. T1 mappingin dilated cardiomyopathy with cardiac magnetic resonance: quantification of diffuse myocardial fibrosis and comparison with endomyocardial biopsy. Eur Heart J Cardiovasc Imaging. 2015;16:210-6.
3. Flett AS, Hayward MP, Ashworth MT, et al. Equilibrium contrast cardiovascular magnetic resonance for the measurement of diffuse myocardial fibrosis: preliminary validation in humans. Circulation. 2010;122:138-44.

4. Mascherbauer J, Marzluf BA, Tufaro C, et al. Cardiac magnetic resonance postcontrast $\mathrm{T} 1$ time is associated with outcome in patients with heart failure and preserved ejection fraction. Circ Cardiovasc Imaging. 2013;6:1056-65.

5. Martinez-Naharro A, Treibel TA, Abdel-Gadir A, et al. Magnetic resonance in transthyretin cardiac amyloidosis. JAm Coll Cardiol. 2017;70:466-77.

6. Sado DM, White SK, Piechnik SK, et al. Identification and assessment of Anderson-Fabry disease by cardiovascular magnetic resonance noncontrast myocardial T1 mapping. Circ Cardiovasc Imaging. 2013;6:392-8.

7. Pan JA, Lee YJ, Salerno M. Diagnostic performance of extracellularvolume, native $\mathrm{T} 1$, and $\mathrm{T} 2$ mapping versus lake Louise criteria by cardiac magnetic resonance for detection of acute Myocarditis: a Meta-analysis. Circ Cardiovasc Imaging. 2018;11:e7598.

8. Puntmann VO, Zeiher AM, Nagel E. T1 and T2 mapping in myocarditis: seeing beyond the horizon of Lake Louise criteria and histopathology. Expert Rev Cardiovasc Ther. 2018;16:319-30.

9. Messroghli DR, Moon JC, Ferreira VM, et al. Clinical recommendations for cardiovascular magnetic resonance mapping of T1, T2, T2* and extracellular volume: A consensus statement by the Society for Cardiovascular Magnetic Resonance (SCMR) endorsed by the European Association for Cardiovascular Imaging (EACVI). J Cardiovasc Magn Reson. 2017;19:75.

10. GranitzM, MotlochLJ, GranitzC, etal. Comparison of native myocardial $\mathrm{T} 1$ and $\mathrm{T} 2$ mapping at $1.5 \mathrm{~T}$ and $3 \mathrm{~T}$ in healthy volunteers: reference values and clinical implications. Wien Klin Wochenschr. 2018; https://doi.org/10.1007/ s00508-018-1411-3.

Publisher's Note Springer Nature remains neutral with regard to jurisdictional claims in published maps and institutional affiliations. 